\title{
Throughput-Delay Tradeoffs in Large-Scale MANETs with Network Coding
}

\author{
Chi Zhang* and Yuguang Fang*† \\ * Department of Electrical and Computer Engineering \\ University of Florida, Gainesville, FL 32611 \\ Email: \{zhangchi@, fang@ece.\}ufl.edu
}

\author{
Xiaoyan Zhu ${ }^{\dagger}$ \\ ${ }^{\dagger}$ National Key Laboratory of Integrated Services Networks \\ Xidian University, Xi'an, China \\ Email: xyzhu@mail.xidian.edu.cn
}

\begin{abstract}
This paper characterizes the throughput-delay tradeoffs in mobile ad hoc networks (MANETs) with network coding, and compares results in the situation where only replication and forwarding are allowed in each node. The schemes/protocols achieving those tradeoffs in an effective and decentralized way are proposed and the optimality of those tradeoffs is established. The scenarios in which network coding can provide significant improvement on network performance are identified under different node mobility patterns (fast and slow mobility). The insights on when and how information mixing is beneficial for MANETs with multiple unicast and multicast sessions are provided. As far as we know, this is the first work characterizing scaling laws of throughput and delay of MANETs with network coding.
\end{abstract}

\section{INTRODUCTION}

One distinct characteristic of wireless mobile ad hoc networks (MANETs) is that, besides transporting data through multi-hop connected paths between the source and destination, packets can also be delivered through the physical mobility of relay nodes which is called store-carry-and-forward paradigm in the literature [1]. Grossglauser and Tse [2] have shown that significant gains in per-node throughput can be obtained by exploiting this paradigm. In particular, they proposed a 2-hop relaying scheme, and showed that it can achieve a constant per-node throughput. The scheme overcomes the throughput bound of $O(1 / \sqrt{n \log n})$ originally established by Gupta and Kumar [3] for a static wireless network, where $n$ is the number of nodes for definitions of the standard asymptotic notation used throughout the paper). Although heavy use of relaying through node mobility allows for higher throughput, it also bears negative side-effects: increased delay. It has been shown in [4], [5] that the 2-hop relaying scheme in [2] yields an extremely large average delay of $\Omega(n)$.

Since both throughput and delay are important network performance metrics from the perspective of an application, significant effort in the last few years has been devoted to understand the throughput-delay relationship in MANETs (refer to Section II-A and the references therein) in the networking research community. An interesting work by Neely and Modiano [5] suggested to utilize redundant packets transmission through multiple opportunistic paths (which are composed of multiple opportunistic links) of a MANET to balance the conflicting requirements on throughput and delay. The basic idea is that the time required for a packet to reach the destination (i.e., end-to-end delay) can be reduced by repeatedly transmitting this packet to many relay nodes of the network, and thus improving the chances that some user

This work was supported in part by the U.S. National Science Foundation under grant CNS-0721744, CNS-0716450, CNS-0626881 and DBI-0529012.

The work of Fang and Zhu is also partially supported by the 111 project under B08038 with Xidian University. holding an original or duplicate version of the packet reaches the destination node. Clearly, the cost of this approach is the decreased throughput since duplicate packets waste scarce opportunities of wireless transmissions. In particular, with i.i.d. mobility, it was shown that for per-node throughput $T(n)=O(1)$, the relaying strategies with replication could yield end-to-end delay $D(n)$ scaling as $\Theta(n \cdot T(n))$ [5].

Previous studies on the scaling laws of MANETs, as discussed above, are all based on the implicit assumption that each node can only perform traditional operations on packets, such as storage, replication and forwarding. Recently, network coding, first introduced by Ahlswede et al. [6] in 2000 , has been widely recognized as a promising primitive operation besides simple replicating and forwarding incoming packets [7]. Using the paradigm of network coding, when a node is scheduled to transmit, it may transmit a "mixed" packet as a result of algebraic operations on its incoming packets to maximize the usefulness of this transmission to all receivers in its transmission range. It is worth noting that a particular useful form of network coding called Random Linear Coding (RLC) was proposed in the literature [8], [9] to independently and randomly mix incoming packets at each node with linear operations, which allows the nodes of the network to achieve the optimal performance in a decentralized fashion.

Intuitively, when RLC instead of replication is used to minimize the end-to-end delay, network congestion can be alleviated and the requirement on buffer size can be relaxed. Therefore, a better throughput-delay tradeoff is expected to be obtained. Since network coding was not taken into consideration in Grossglauser and Tse's original work [2] and the related work [4], [5], [10] that followed, an interesting question raised naturally is how much benefit network coding can provide to the network performance of MANETs compared to when only simple replication and forwarding are allowed for relay nodes. Answering this question will help us better understand not only the benefits and limitations of network coding in wireless networks but also the fundamental tradeoffs determining MANET's performance.

In this paper we conduct a thorough study on the scaling laws governing MANETs. We characterize the throughputdelay tradeoffs with respect to different node mobility patterns. We identify scenarios in which network coding can provide significant improvement on network performance. Note that our work differentiates MANETs from static wireless networks by the roles network coding plays, because previous work showed that network coding could only provide constant improvement on the throughput of static wireless networks (cf. Section II-C and the references therein). We also provide insights on when and how information mixing is beneficial and propose algorithms to show that these benefits can be achieved in an effective and decentralized fashion. 


\section{BACKGROUND AND RELATED WORK}

\section{A. Scaling Laws of MANETs without Network Coding}

Seminal work of Gupta and Kumar [3] initiated the investigation on how the throughput of wireless networks scales with $n$, the number of nodes. Under the assumption that nodes with common transmission range are randomly distributed, it is shown that per-node throughput for static wireless networks scales as $\Theta(1 / \sqrt{n \log n})$. Note that [3] implicitly used a fluid model for establishing throughput scaling. Later work by Kulkarni and Viswannath [11] consolidated the result of [3] with an explicit constant packet size model. In [12], with percolation theory, Franceschetti et al. showed that the $\Theta(1 / \sqrt{n})$ per-node throughput is achievable if each node can adjust its transmission range (through power control), however, the throughput vanishing problem for large-scale $(n \rightarrow \infty)$ static wireless networks still remains. In [2], Grossglauser and Tse showed that the mobility of the nodes in a MANET can be exploited to overcome this problem. The 2-hop relaying scheme they proposed achieves a constant per-node throughput at the cost of a large delay on the order of $n$ [4], [5]. This result reveals the possibility of trading larger delay for higher throughput or lower throughput for smaller delay in MANETs. Since then, a flurry of research activities have tried to characterize the throughput-delay relationship with respect to node mobility, e.g., [4], [5], [13]-[20].

In general, there are two ways to trade throughput for delay in the literature. Kleinrock et al. [21] may be the first to find that delay can be reduced by increasing the transmission radius of each relay node, at the expense of reducing the number of simultaneous transmissions the network can support, which leads to a lower throughput. Similar transmission radius scaling techniques have appeared in [4], [14]-[20]. Another approach, which improves delay via redundant packet transfers is considered in [5], [22]. In this paper, we follow this approach, adopting redundant strategy and comparing it with network coding for the following reasons:

- First of all, the assumption that transmission ranges can scale with $n$, the number of nodes, is impractical for large-scale MANETs. To obtain the scaling law of MANETs, we usually require $n$ tending to infinity, which is equivalent to assuming $\sqrt{A_{n}} \rightarrow \infty$ for extended network model, where $A_{n}$ is the area of the network (cf. Section III-A). In general, wireless device is power limited, rendering it impossible to require the transmission range reaching the order of $\sqrt{A_{n}}$.

- Second, tradeoffs theoretically analyzed using the first means mentioned above are mainly based on fluid model, in which the packets are allowed to be arbitrarily small as $n \rightarrow \infty$ (e.g., [4], [14]-[17], [19], [20]). On the other hand, tradeoffs obtained through the second approach assume constant packet size model ${ }^{1}$, where the packet size remains constant, i.e., does not scale down with $n$ (e.g., [5]). We prefer the constant packet size model since in reality, packet size does not change when more nodes are added to the network. Furthermore, fluid model cannot be applied to scenarios with network coding, since every coded packet includes a "code vector" of at least constant size for successful decoding.

\footnotetext{
${ }^{1}$ Note that with the additional constraint that the packet size remains constant, the throughput-delay tradeoff can be no better than that in the fluid model, and the analysis of constant packet size model is much harder than that of fluid model [18]. Throughout the paper, our results on scaling laws of MANET with or without network coding are all based on constant packet size model (cf. Section III-A) for the rigor of theoretical analysis.
}

- Finally, in this paper we are interested in examining pure gains introduced by network coding in MANETs. Replication strategies can be replaced by network coding, which provides a good chance for comparison. Transmission radius scaling techniques, however, are orthogonal to network coding, and should be studied separately.

We would like to point out that all the results discussed above are based on the implicit assumption that only storageand-forwarding (without network coding) is allowed in each node, while in this paper we seek to understand whether network coding indeed affects the throughput-delay tradeoffs in MANETs.

\section{B. Network Coding Applications in Wireless Networks}

At the very beginning, research on network coding mainly focused on multicast scenario. In their pioneering theoretical work, Alswede et al. [6] showed that the min-cut throughput of a multicast session on a directed graph can be achieved, provided that one allows network coding, i.e., encoding at the intermediate nodes of the network. Conversely, it is generally not possible to achieve this communication rate if one allows only routing or copying packets at the intermediate nodes of the network. Shortly afterwards, Li, Yeung, and Cai [23] showed that it is sufficient for the encoding functions at the interior nodes to be linear. Subsequent work by Jaggi et al. [8] and Ho et al. [9] showed that the linear encoding functions can be designed randomly and independently at each node, which leads to a particular useful form of network coding, the RLC. Since RLC operates in a decentralized fashion [24], [25] which is extremely suitable for MANETs where centralized control is almost impossible or costly, we concentrate on RLC throughout the paper. Performance of RLC for local and global broadcast in wireless networks has been extensively studied in the literature, e.g., [7], [26]-[30].

For practically more important case of multiple unicast, we can only ascertain that it is totally different from multicast cases. For example, $\mathrm{Li}$ and $\mathrm{Li}$ conjectured in [31] that for undirected network with multiple unicast sessions, network coding does not help much on throughput. A deep understanding on achievable throughput for multiple unicast sessions in a network is still an open problem. In general, it is not clear whether network coding should be performed, and if it should, what the strategy must be [7]. One of our main contributions in this paper is that we analytically address this problem and show that, although RLC still cannot improve the order of throughput in MANETs, it changes the achievable throughput-delay tradeoffs significantly, which we believe will help improve our understanding of the theoretical limits on the benefits of network coding and on how to achieve them for MANETs with multiple unicast sessions.

The idea that, when RLC is allowed in intermediate nodes, compared to replication strategies [5], [22], larger throughput can be achieved with the same delay and smaller sizes of node buffers, was perhaps first explicitly developed in the work [32] by Zhang et al., where a simulation-based study of the benefit of RLC in one unicast communication is also presented. The recent work by $\mathrm{Lin}$ and $\mathrm{Li} \mathrm{[33]} \mathrm{gives} \mathrm{a} \mathrm{rigorous}$ analysis of this idea based on ordinary differential equations. To our knowledge, [32] and [33] are the closest to our work in terms of understanding the relationships between throughput and delay with network coding. However, our work has the following advantages:

- First of all, instead of explicitly modeling nodes' spatial distributions as in this paper, the mobility of nodes 
in [32] and [33] is modeled with meeting times of any pair of nodes, to simplify the analysis. The problem is that, the most important feature of wireless transmission, i.e., interference, is not included in their modeling. It is nevertheless still reasonable for [32] and [33], since the authors are mainly interested in delay tolerant networks, where nodes are assumed to be sparsely distributed and interference from simultaneous transmissions can be ignored. However, it is obviously not suitable for the study of general MANETs.

- Second, the traffic pattern considered in our paper is more practical. The number of unicast sessions supported in this paper is $\Theta(n)$, while only one unicast or broadcast session is assumed in [32] and [33].

- Next, only epidemic routing and its replacement of network coding are considered in [32] and [33], while in our work, several alternatives are considered and different algorithms are developed, which achieve throughputdelay pairs on different orders of $n$. Therefore, we obtain a complete characterization of tradeoffs in MANETs.

- Most importantly, explicit expressions of network performance or tradeoffs are obtained in our paper for the first time, which provides insights on the degree of scalability of MANETs with network coding.

\section{Scaling Laws of Wireless Networks with Network Coding}

Scaling laws governing wireless networks with network coding have only been investigated in the limited scenarios in the literature recently. The delay gains and reliability benefit (measured in the reduced number of transmissions) of network coding in unreliable wireless networks were characterized in [34], [35] and [36], respectively. However, these results are for one multicast session with one-hop transmission or stable network topology. For multiple unicast scenario, Liu et al. [37], [38] and Keshavarz-Haddadt et al. [39] showed that for static wireless networks, network coding and broadcasting at most provide a constant-factored improvement in the throughput, compared to Gupta and Kumar's $\Theta(1 / \sqrt{n \log n})$ per-node throughput [3]. As far as we know, the scaling laws for throughput and delay are still unexploited for MANETs in the literature. More importantly, our results show that, network coding can provide significant improvement on network performance when mobility is utilized, which is impossible in static wireless networks [37]-[39]. We believe it reveals the intrinsic difference between MANETs and static wireless networks.

\section{MANET MODELS AND DEFINITIONS}

\section{A. Network Models}

Random network model for MANETs: Consider an ad hoc network where $n$ nodes are distributed uniformly at random in a square area of $A_{n}$. The square is assumed to be a torus ${ }^{2}$, i.e., the top and bottom edges are assumed to touch each other and similarly the left and right edges also are assumed to touch other. We consider a multiple $(n)$ unicast scenario in which each node $i \in\{1,2, \cdots, n\}$ is a source node for one unicast session, and a destination node for another unicast session. Suppose that the source node $i$ has data intended for destination node $d(i)$. We assume that each source node has an infinite stream of packets to send to its destination.

\footnotetext{
${ }^{2}$ We assume the torus to avoid edge effects, which otherwise complicates the analysis. We note, however, that the results in the paper hold for square, disk or any other shapes of practical interests.
}

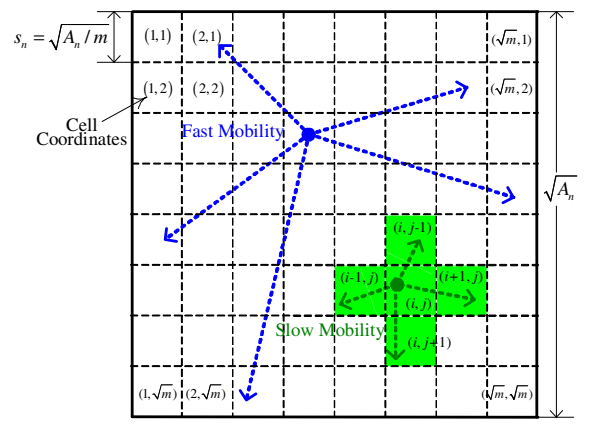

Fig. 1. Fast and slow mobility models for MANETs.

The source-destination (S-D) association does not change with time, although the nodes themselves move.

Mobility models: The torus is divided into $m=\Theta(n)$ square cells of area $A_{n} / m$ each, resulting in a two-dimensional $\sqrt{m} \times$ $\sqrt{m}$ discrete torus ${ }^{3}$, see Fig. 1 for an illustration. The initial position of each node is equally likely to be any of the $m$ possible cells independent of others. We assume the time is slotted and we study the following mobility models in this paper:

- Fast mobility model (i.i.d. mobility model): At each time slot, nodes randomly choose a new cell location independently and identically (i.i.d.) distributed over all cells in the network. This model captures the situation when mobile user moves so quickly that its position is almost independent from time to time. With this assumption, the network topology dramatically changes in every time slot, so that the network behavior cannot be predicted and fixed routing algorithms cannot be used. This mobility model is also used in [5], [14], [16], [19], [20].

- Slow mobility model (random walk model): Let a node be in cell $(i, j) \in\{1, \cdots \sqrt{m}\}^{2}$ at time slot $t$, then, at time slot $t+1$, the node is equally likely to be in the same cell $(i, j)$ or any of the four adjacent cells $\{(i-1, j),(i+1, j),(i, j-1),(i, j+1)\}$, where addition and subtraction are modulo $\sqrt{m}$. So each node in fact independently performs a simple random walk on the two-dimensional $\sqrt{m} \times \sqrt{m}$ discrete torus. Note that this model implicitly sets an upper bound on the velocity of nodes as $\sqrt{2 A_{n} / m}$. Therefore, it is a suitable model for capturing real motion of nodes with slow mobility. Similar mobility model is also adopted in [4], [17]-[19].

Model for successful transmission: For characterizing the condition for a successful transmission, we adopt the protocol model as defined in [3]. We assume that all nodes use a common range $r_{c}$ for their transmissions, and a transmission from node $i$ to node $j$ is successful if and only if $d_{i j} \leq r_{c}$ and $d_{k j} \geq(1+\Delta) r_{c}$ for any other simultaneous transmitter, say node $k$. Here, $d_{i j}$ is the distance between nodes $i$ and $j$, and $\Delta$ is a positive constant independent of $n$. During a successful transmission, nodes send data at a constant rate of $W$ bits per second. In the other commonly used model of successful transmission, namely, the physical model, a transmission is successful if the SINR is greater than some constant. It is well known [3], [4] that with a fading factor $\alpha>2$, the protocol model is equivalent to the physical model. Therefore, we prefer the use of the protocol model in this paper for a cleaner presentation of the key ideas.

Concurrently transmitting cells: Now we define the transmission range and schedule. We choose $r_{n}$ in such a way

\footnotetext{
${ }^{3}$ For simplicity, assume $\sqrt{m}$ is an integer.
} 


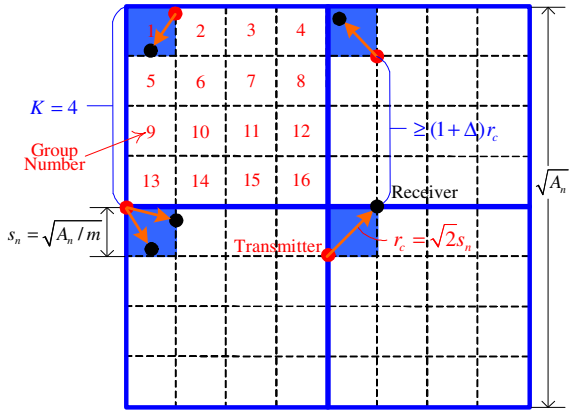

Fig. 2. Cell transmission scheduling. Here is an illustration of the cells being divided into $K^{2}$ groups for the case of $K=4$, i.e., 16 groups. All the blue cells which are in group 1 transmit in the same timeslot. In the next timeslot all the cells in group 2 transmit and so on.

that any node in a cell can always directly transmit to any other node in the same cell using the smallest common range of transmission. Obviously, $r_{c}=\sqrt{2} s_{n}=\sqrt{2 A_{n} / m}=$ $\Theta\left(\sqrt{A_{n} / n}\right)$. Time is slotted for packetized transmission. We assume only $O(1)$ packets can be transmitted per cell per timeslot, i.e., our analysis is explicitly based on constant packet size model (see [18] and Section II-A for detailed discussion). We adopt the cell scheduling scheme shown in Fig. 2, which has the following Proposition [11].

Proposition 1: Under the Protocol model, there exists an interference-free schedule such that each cell becomes active regularly once in $K^{2}$ timeslots and it does not interfere with any other simultaneously transmitting cells. Here $K$ depends only on $\Delta$, and is independent of $n$.

Extended network model: We are particularly interested in asymptotic properties of MANETs, which hold with high probability $^{4}$ for large-scale MANETs. Therefore, we need often take limits as $n \rightarrow \infty$. When the region area $A_{n}$ is fixed, it corresponds to a dense network model [3], [40], because the density of the network $d=n / A_{n}$ also tends to infinity as $n$. Another widely used model is the extended network model [41], [42], in which both the number of nodes and the area of the region $A_{n}$ go to infinity while $d$ is kept constant. Both models are widely used in the literature and we will focus on the latter one. In the extended network model, $A_{n}=n / d=$ $\Theta(n)$, and correspondingly $r_{c}=\Theta\left(\sqrt{A_{n} / n}\right)=\Theta(1)$. This is more practical, since power constraint on wireless devices does not change when more nodes are added to the network. We note that, however, results obtained in this paper can be easily extended to dense network model.

\section{B. Network Performance Metrics}

Definition of throughput: A throughput $\lambda>0$ is said to be feasible/achievable if every node can send at a rate of at least $\lambda$ bits per second to its chosen destination. We denote by $T(n)$, the maximum feasible throughput w.h.p. Given a scheme $\Pi$, let $M_{\Pi}(i, t)$ be the number of packets from source node $i$ that destination node $d(i)$ receives in $t$ timeslots under scheme $\Pi$, for $1 \leq i \leq n$. Note that this could be a random quantity for a given realization of the network. Define the long term throughput of S-D pair $i$, denoted by $\lambda_{\Pi}^{i}(n)$, to be

$$
\lambda_{\Pi}^{i}(n)=\liminf _{t \rightarrow \infty} \frac{1}{t} M_{\Pi}(i, t) .
$$

Scheme $\Pi$ is said to have throughput $T_{\Pi}(n)$ if

$$
\lim _{n \rightarrow \infty} \mathbb{P}\left(\lambda_{\Pi}^{i}(n) \geq T_{\Pi}(n) \text { for all } i\right)=1 .
$$

${ }^{4}$ We say that an event occurs with high probability (w.h.p.) if its probability tends to 1 as $n \rightarrow \infty$.
We allow randomness in schemes and, as a result, random quantities above are in the joint probability space including both the random network of size $n$ and the scheme $\Pi$. Note that when network coding is utilized in scheme $\Pi, M_{\Pi}(i, t)$ is the number of successfully decoded packets received by the destination $d(i)$ of S-D pair $i$ in $t$ timeslots under scheme $\Pi$.

Definition of delay: The delay of a packet is the time it takes the packet to reach the destination after it leaves the source. We do not take queueing delay at the source into account, since our interest is in the network delay. Let $D_{\Pi}^{i}(j)$ denote the delay of packet $j$ of S-D pair $i$ under scheme $\Pi$, then the sample mean of delay for S-D pair $i$ is

$$
\bar{D}_{\Pi}^{i}=\limsup _{k \rightarrow \infty} \frac{1}{k} \sum_{j=1}^{k} D_{\Pi}^{i}(j) .
$$

The average delay over all S-D pairs for a particular realization of the random network is then $\bar{D}_{\Pi}=\frac{1}{n} \sum_{i=1}^{n} \bar{D}_{\Pi}^{i}$. The delay for a scheme $\Pi$ is the expectation of the average delay over all S-D pairs and all random network configurations, i.e.,

$$
D_{\Pi}(n)=\mathbb{E}\left[\bar{D}_{\Pi}\right]=\frac{1}{n} \sum_{i=1}^{n} \mathbb{E}\left[\bar{D}_{\Pi}^{i}\right] .
$$

When network coding is utilized, we consider the delay of getting original packets. When an original packet $m_{i}$ belongs to the generation $M$, the delay of $m_{i}$ under scheme $\Pi$ is the time from the first packet belonging to $M$ leaves the source to the original packet $m_{i}$ has been decoded in the destination.

\section{Throughrut-Delay TradeofFs WithouT Network COding: Schemes AND Results}

In this section, we give a brief overview of the redundancybased schemes as presented in [5] and establish the throughput-delay tradeoffs in MANETs without network coding. Some of the discussions presented here directly build on results already established in [5]. They are included here for completeness and comparison purposes .

We first describe three relay schemes with different redundancy proposed in [5] from a unified point of view.

\section{Three Redundancy-Based Schemes Proposed in [5]:}

We can control the transmission redundancy of each packet with two methods: the number of hops each packet will take from source to destination, and the total number of copies (replicas) of each original packet in the network. The three schemes, namely, 2-hop relay without replicas, 2-hop relay with $k_{1}$ replicas, and multi-hop relay with $k_{2}$ replicas represent different combinations of the two methods.

Each scheme has two parts: (1) scheduling of active cells; (2) scheduling of transmission in an active cell.

The three schemes have the same cell scheduling policy (Part (1)) as follows:

- Each cell becomes active once in every $K^{2}$ timeslots as discussed in Proposition 1.

- In an active cell, transmission is always between two nodes within the same cell.

In every active cell with at least two nodes, intra-cell transmission scheduling (Part (2)) is needed.

- For 2-hop relay schemes, each packet at most takes two hops from source to destination. The difference is that, for 2-hop relay without replicas, packets are not duplicated and are held by at most one node (source or relay) at any timeslot, while for 2-hop relay with $k_{1}$ replicas, the source will send $k_{1}$ replicas to distinct nodes as relays. 
When destination nodes receive packets from relays, they will first tell relays which packet they are looking for before the transmission begins (using the handshake).

- Multi-hop relay with $k_{2}$ replicas is just another type of flooding scheme, which transmits $k_{2}$ replicas of each packet, and places no constraints on the number of hops. At every timeslot in each active cell, the oldest packet will be selected to send to all nodes in the cell.

Now, we analyze the performance of the schemes described above. First of all, we give the lower bounds on delays under fast mobility model as follows [5].

Theorem 1: Under fast mobility model, $D(n)=\Omega(\log n)$ for any scheme and $D(n)=\Omega(\sqrt{n})$ for any 2-hop relay scheme.

Remark 1: To achieve the optimal throughput with the minimal delay given above, parameters like $k_{1}$ and $k_{2}$ in the proposed schemes should be carefully chosen under different mobility models. It has been shown in [5] that under fast mobility model, $k_{1}=\Theta(\sqrt{n})$ is enough for 2-hop relay scheme to achieve the minimal delay $\Theta(\sqrt{n})$. Further increasing redundancy $k_{1}$ will only reduce throughput without decreasing delay. Following the same argument, we have the optimal $k_{2}=\Theta(\log n)$ for flooding scheme. These lead to the following Theorem.

Theorem 2: Assuming infinite buffer space at each node, throughput-delay tradeoffs achieved by the three redundancybased schemes proposed in [5] for MANETs under fast mobility model can be summarized in the following table.

\begin{tabular}{|c|l|l|}
\hline scheme & throughput & delay \\
\hline 2-hop relay without replicas & $\Theta(1)$ & $\Theta(n)$ \\
2-hop relay with $k_{1}$ replicas & $\Theta(1 / \sqrt{n})$ & $\Theta(\sqrt{n})$ \\
multi-hop relay with $k_{2}$ replicas & $\Theta\left(\frac{1}{n \log n}\right)$ & $\Theta(\log n)$ \\
\hline
\end{tabular}

Proof: The proof of Theorems 1 and 2 is similar to the proof of Theorems 3, 6, 7 and 8 in [5], with minor differences caused by our use of the protocol model and cell scheduling scheme, ignoring queueing delays at source nodes. Due to space constraints, we do not repeat the proof here.

Next, we consider the throughput-delay tradeoffs under slow mobility model. We first show that the tradeoff with slow mobility is dramatically different from the one with fast mobility by the following theorem.

Theorem 3: Under slow mobility model, $D(n)=\Omega(\sqrt{n})$ for any scheme and $D(n)=\Omega(\sqrt{n} \log n)$ for any 2-hop relay scheme.

Proof: From random walk model, node speed is upper bounded by $\sqrt{2 A_{n} / m}=O(1)$ and the transmission range $r_{c}=\Theta(1)$. Therefore, information propagation speed will be no larger than $\Theta(1)$ per timeslot. It can be shown that the distance between the initial positions of S-D pair is $\Omega\left(\sqrt{A_{n}}\right)=\Omega(\sqrt{n})$ w.h.p. [11]. Hence, the expected delay is at least $\Omega(\sqrt{n})$ timeslots. For 2-hop relay cases, see our technical report [43] for a complete proof.

Theorem 4: Assuming infinite buffer space at each node, throughput-delay tradeoffs achieved by the three redundancybased schemes proposed in [5] for MANETs under slow mobility model can be summarized in the following table.

\begin{tabular}{|c|l|l|}
\hline scheme & throughput & delay \\
\hline 2-hop relay without replicas & $\Theta(1)$ & $\Theta(n \log n)$ \\
2-hop relay with $k_{1}$ replicas & $\Theta\left(\frac{1}{\sqrt{n} \log n}\right)$ & $\Theta(\sqrt{n} \log n)$ \\
multi-hop relay with $k_{2}$ replicas & $\Theta\left(\frac{1}{n \sqrt{n}}\right)$ & $\Theta(\sqrt{n})$ \\
\hline
\end{tabular}

Proof: The proof for the performance of 2-hop relay without replicas can be found in [4], [17]. The proof for the other two schemes are not reported in the literature. We complete the proof of this theorem in our technical report [43]. Note that the performance above is achieved with $k_{1}=\Theta(\sqrt{n \log n})$ and $k_{2}=\Theta(\sqrt{n})$, respectively.

\section{Throughrut-Delay TradeofFs With Network CODING: SCHEMES AND RESULTS}

We first review RLC used in our network coding based schemes. This bears exactly the same setup as in [25]. Then we describe the schemes developed for analyzing tradeoffs in MANETs with network coding, and identify scenarios in which RLC improves network performance of MANETs.

\section{A. Network Coding Operation}

Random linear coding (RLC) is applied to a finite set of $k$ original packets (i.e., $M=\left\{m_{1}, m_{2}, \cdots, m_{k}\right\}$ ), that is called a generation. Each packet is viewed as an $r$-dimensional vector over a finite field, $\mathbb{F}_{q}$ of size $q$, i.e., $m_{i} \in \mathbb{F}_{q}^{r}, i=1,2, \cdots, k$. If the packet size is $m$ bits, this can be done by viewing each packet as an $r=\left\lceil m / \log _{2}(q)\right\rceil$-dimensional vector over $\mathbb{F}_{q}$ (instead of viewing each packet as an $m$-dimensional vector over binary field). Typically, $\mathbb{F}_{2^{8}}$ (i.e., $\mathbb{F}_{256}$ ) is used. All the additions and multiplications in the following description are assumed to be over $\mathbb{F}_{q}$. We assume that all the $k$ packets in $M$ are linearly independent. During the execution of a RLC based relay scheme, the destination node of $M$ collects linear combinations of the packets in $M$. Once there are $k$ independent linear combinations at a node, it can recover all the original packets in $M$ successfully.

Now, consider a certain timeslot $t$. Let $S_{v}(t)$ and $S_{u}(t)$ denote the set of all the coded packets (each coded packet is a linear combination of the packets in $M$ ) at node $v$ and $u$, respectively, at the beginning of the timeslot $t$. More precisely, if coded packet $f_{l} \in S_{v}(t)$, where $l=1,2, \cdots,\left|S_{v}(t)\right|$, then $f_{l} \in \mathbb{F}_{q}^{r}$ has the form $f_{l}=\sum_{i=1}^{k} \alpha_{l i} \cdot m_{i}, \alpha_{l i} \in \mathbb{F}_{q}$. The scheme ensures that $a_{l i}$ 's are known to node $v$ by appending each packet $f_{l}$ with a "code vector", which will be explained a little later. Let $S_{v}(t)^{-}$and $S_{u}(t)^{-}$denote the subspaces spanned by the coded packets in $S_{v}(t)$ and $S_{u}(t)$, respectively. If $S_{v}(t)^{-} \nsubseteq S_{u}(t)^{-}$, we say node $v$ has useful information about $M$ for $u$. In timeslot $t$, if node $v$ is scheduled by the scheme to transmit a packet related to $M$ to node $u, v$ first checks if it has useful information for $u$. If so, $v$ transmits a "random" coded packet with payload $f_{\text {new }} \in \mathbb{F}_{q}^{r}$ to $u$, where

$f_{\text {new }}=\sum_{f_{l} \in S_{v}(t)} \beta_{l} \cdot f_{l}, \beta_{l} \in \mathbb{F}_{q}$ and $\mathbb{P}\left(\beta_{l}=\beta\right)=\frac{1}{q}, \forall \beta \in \mathbb{F}_{q}$.

It is easy to check that $f_{n e w}$ is still a linear combination of the $k$ original packets, and can be written as $f_{\text {new }}=\sum_{i=1}^{k} \theta_{i} \cdot m_{i}$ where $\theta_{i}=\sum_{f_{l} \in S_{v}(t)} \beta_{l} \cdot \alpha_{l i} \in \mathbb{F}_{q}$. For decoding purposes, the vector $\left(\theta_{1}, \theta_{2}, \cdots, \theta_{k}\right) \in \mathbb{F}_{q}^{r}$, called code vector, will be appended to $f_{\text {new }}$, and sent as overhead. This overhead clearly requires a padding of additional $k \log _{2}(q)$ bits. If the packet size $m \gg \log _{2}(q)$, which would be the case under our constant packet size model, then the overhead required by the RLC based scheme can be ignored in our analysis. ${ }^{5}$

\footnotetext{
5 More precisely, the constant packet size model for original packets means that the packet size scales as $\Theta(\log n)$ bits, since it needs to carry the ID of the destination node with $\Theta(\log n)$ bits. For a fair comparison, we require that $k=O(\log n)$ for the coded packets throughout the paper. Therefore the overhead introduced by the code vector will not change the order of our results on $T(n)$ and $D(n)$ for RLC-based schemes.
} 
We say that $v$ sends an innovative coded packet $f_{\text {new }}$ to $u$, if $f_{n e w}$ can increase the dimension of the subspace $S_{u}(t)^{-}$, i.e., $\operatorname{dim}\left(S_{u}(t)^{-}\right)$. Note that $\operatorname{dim}\left(S_{u}(t)^{-}\right) \leq k$ in general and if $\operatorname{dim}\left(S_{u}(t)^{-}\right)=k$, node $u$ can recover all the $k$ original packets at once. We now recall the following key result about RLC, which says that $f_{\text {new }}$ will be an innovative coded packet for $u$ with probability no less than $1-\frac{1}{q}$.

Proposition 2: (Lemma 2.1 in [25]) Let $S_{u}(t)^{+}=S_{u}(t) \cup$ $\left\{f_{\text {new }}\right\}$ be the subspace spanned by the code vectors in $u$ at the end of timeslot $t$, i.e., after receiving a coded packet $f_{\text {new }}$ from $v$ according to the RLC based scheme described as above. Then,

$\mathbb{P}\left(\operatorname{dim}\left(S_{u}(t)^{+}\right)>\operatorname{dim}\left(S_{u}(t)^{-}\right) \mid S_{v}(t)^{-} \nsubseteq S_{u}(t)^{-}\right) \geq 1-\frac{1}{q}$

\section{B. RLC-Based Relay Schemes}

In this subsection, we describe RLC-based relay schemes with different routing strategies, which will be used later to exploit throughput-delay tradeoffs in MANETs.

We first introduce the concept of big generation. In what follows, when we say that the source node groups $k=$ $\omega(\log n)$ original packets into one big generation, we in fact separate these $k$ packets into $k / \Theta(\log n)$ generations, each with $\Theta(\log n)$ packets. When the destination node tries to decode one original packet, it first needs to collect $\Theta(k)$ coded packets from the big generation (with $\Theta(\log n)$ coded packets from each generation). Therefore the overhead introduced by RLC is ignorable in our analysis (cf. footnote 5).

\section{Schemes 1: 2-hop Relay with RLC}

(1) $k$ original packets in each source node will be grouped into one (big) generation. Each source will send $m=(1+\epsilon) k$ coded packets for each (big) generation, where $\epsilon$ is a constant. (2) Coded packets for each generation will have the same timestamp $t_{p}$. The value of $t_{p}$ is the time the first coded packet of that generation leaves the source. All coded packets of a generation will be deleted from the relay buffer at the timeslot $t$ if $t-t_{p}>t h_{p}$, where the threshold $t h_{p}$ depends on $D(n)$ of the scheme and will be sufficiently larger than $D(n)$.

(3) Each cell becomes active once in every $K^{2}$ timeslots as discussed in Proposition 1. In an active cell, transmission is always between nodes within the same cell.

(4) For an active cell with at least two nodes, a random transmitter-receiver pair is selected, with uniform probability over all possible node pairs in the cell. With probability $1 / 2$, the transmitter is scheduled to operate in either "Source-toRelay" or "Relay-to-Destination" mode, described as follows:

- Source-to-Relay Mode: The transmitter sends a coded packet of its current generation, and does so upon every transmission opportunity while it is in source-to-relay mode until $m$ coded packets have been delivered to distinct nodes. If all other nodes in the cell already have one coded packet for that generation, the source will begin to transmit coded packets from the next generation. Every node stores a single packet per S-D pair per generation. When the node receives a new packet, a relay linearly combines the incoming packet with the stored one, and replaces the stored packet with the result. Note that the nodes operate in broadcast mode, i.e., every node will hear every transmission in its range, and update the packet storage as described above.

- Relay-to-Destination Mode: If the designated transmitter has a coded packet in its relay buffer for the destination node, and the rank of coded packets of that generation in the receiver is smaller than $k$, the coded packet is transmitted to the designated receiver.

Remark 2: Since $m>k$, we need a mechanism to stop unnecessary relay of coded packets of a generation when it is already decoded in the destination. Here we use a proactive stopping mechanism, i.e., the timestamp of each generation, since we can bound the delay of the scheme. In the analysis part presented later, we will show that $k=\Theta(n)$, and $D(n)$ for this scheme is also $\Theta(n)$ for fast and slow mobility models. Therefore, $t h_{p}$ should be larger than $\Theta(n)$. More complicated reactive stopping mechanisms (cf. [33] and the references therein) can be adopted to enhance the efficiency of the scheme in practice. However, we follow the simplest design for analytical tractability of the scheme.

\section{Schemes 2: Multi-hop Relay with RLC}

(1) $k$ original packets in each source node will be grouped into one (big) generation. Each source will send $m=(1+\epsilon) k$ coded packets for each generation, where $\epsilon$ is a constant. Two timestamps for each generation are used. One is called the generating time $t_{g}$, based on the time for $k$ original packets to be grouped into a generation in the source. Another is called transmission time $t_{p}$, based on the time the first coded packet of that generation is transmitted by the source.

(2) Each cell becomes active once in every $K^{2}$ timeslots as discussed in Proposition 1. In an active cell, transmission is always between nodes within the same cell.

(3) For an active cell with at least two nodes, perform the following: among all packets contained in at least one node of the cell and which have useful information for some other node in the same cell, choose the packet with the smallest generating time $t_{g}$. If there are ties, choose the packet from the S-D pair $i$ which maximizes $\left(t_{g}+i\right) \bmod n$. Transmit this packet to all other nodes in the cell. If the selected packet is in the source, then the source will transmit the linear combination of its $k$ original packets of the same generation, instead of a particular packet belonging to that generation.

(4) Every node stores a single packet per S-D pair per generation. When the node receives a new packet, a relay linearly combines the incoming packet with the stored one, and replaces the stored packet with the result.

(5) All coded packets of a generation will be deleted from the relay buffer at the timeslot $t$ if $t-t_{p}>t h_{p}$, where the threshold $t h_{p}$ depends on $D(n)$ of the scheme and should be sufficiently larger than $D(n)$.

Remark 3: The generating timestamp $t_{g}$ is used to construct a flooding scheme for one particular S-D pair where all $n \mathrm{~S}-\mathrm{D}$ pairs are active and share the network resource. It is easy to see that the packets from the oldest generation that has not been delivered to all nodes will dominate the transmissions over the whole network very quickly. The long-term fairness between all S-D pairs is guaranteed since in the case of ties, packets from S-D pair $i$ are given top priority in every $n$ timeslots. Also note that, since at one particular timeslot, only one generation from one S-D pair dominates the whole network, the number of packets each relay needs to store in step (4) is 1, i.e., just for one generation w.h.p. Another timestamp $t_{p}$ used here has the same functionality as the previous scheme. The threshold $t h_{p}$ should be larger than $D(n)$, scaling as $\Theta(\log n)$ and $\Theta(\sqrt{n})$, respectively, for fast and slow mobility models.

\section{Main Results for RLC-Based Schemes}

In this subsection, we summarize the performance of the above schemes under different mobility models. Here, we 


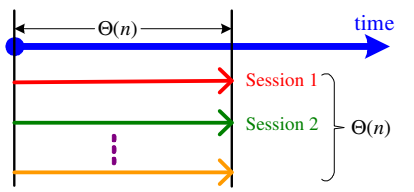

(a) 2-hop relay with RLC coding

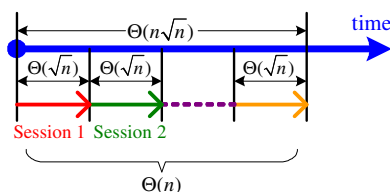

(b) Multi-hop relay with RLC coding
Fig. 3. Timetables for different RLC-based schemes under slow mobility model.

focus on the intuition and explanation of these results. Proofs of theses results will be given in the next Section.

Theorem 5: When 2-hop relay with RLC scheme is used and $k=\Theta(n)$, we have $T(n)=\Theta(1)$ and $D(n)=\Theta(n)$ for fast and slow mobility models.

Remark 4: Compare to Theorems 2 and 4, it is easy to see that, RLC provides delay improvement $\Theta(\log n)$ under slow mobility model. No gain is found under fast mobility model. It is not surprising, since 2-hop relay with RLC scheme is used to replace 2 -hop relay without replicas, and we know that in the latter, there is no duplicated packets in order to maximize the throughput. Thus we cannot expect any gains when network coding is used. The gain $\Theta(\log n)$ of delay under slow mobility model comes from the lower information propagation speed, and the mixing of packets increase this speed by guaranteeing that every packet the destination received from relay nodes will contribute some information for the decoding of the packet from the same generation. For fast mobility model, this benefit vanishes since the information propagation speed is high enough, and the delay for waiting $k$ coded packets for decoding dominates the whole delay.

Theorem 6: When multi-hop relay with RLC scheme is used, under fast mobility model with $k=\Theta(\log n)$, we have $T(n)=\Theta(1 / n)$ and $D(n)=\Theta(\log n)$. Under slow mobility model with $k=\Theta(\sqrt{n})$, we have $T(n)=\Theta(1 / n)$ and $D(n)=\Theta(\sqrt{n})$.

Remark 5: Under fast and slow mobility models, multihop RLC-based schemes always provide significant gains compared to flooding schemes. We can see that the RLC-based scheme can achieve minimal delay, with an improved delayconstrained throughput. The intuition is that, when flooding is used, there exist enough opportunities to enhance performance by replacing replicas with more intelligent coding.

Fig. 3 compares timetables of 2-hop and multi-hop RLCbased relay schemes. It can be found that in 2-hop relay schemes, multiple sessions operate in a parallel fashion, while in multi-hop relay schemes, they operate in a sequential fashion. Therefore, at each timeslot, for 2-hop relay schemes, traffic pattern is still multiple unicasts. Recall our discussion in Section II-B, for multiple unicasts, we seldom find gains from network coding. While for multi-hop relay schemes, at each timeslot, traffic pattern looks more like one broadcast session, where gains from network coding are naturally expected.

Remark 6: Also notice that, multi-hop relay schemes can be divided into multiple phases, and in each phase, relaying for one generation from one S-D pair will dominate the network, which is in fact a type of information flooding in this phase (refer to Fig. 3(b) for illustration). The result is that in each phase, packets from one generation will be broadcasted to the whole network, and if the other $n-1$ nodes are receivers, they can all decode the original packets in that generation at the end of that phase. So it guarantees that multi-hop relay with RLC coding can support all-to-all traffic pattern ( $n$ broadcast sessions) with the same performance. Note that this also means that the same network performance can be achieved for any $n$ multicast sessions (since receivers in this case are just a subset of receivers in the broadcast case). From Theorem 6, we can easily obtain the following corollary on the performance of multiple broadcasts and multicasts with network coding.

Corollary 1: For all-to-all communications or any multicasts with $n$ sources, when multi-hop relay with RLC scheme is used, under fast mobility model with $k=\Theta(\log n)$, we have $T(n)=\Theta(1 / n)$ and $D(n)=\Theta(\log n)$. Under slow mobility model with $k=\Theta(\sqrt{n})$, we have $T(n)=\Theta(1 / n)$ and $D(n)=\Theta(\sqrt{n})$.

In [30], Fragouli et al. designed an RLC-based scheme based on results from [25]. For all-to-all communications, they showed that their scheme achieves $T(n)=\Theta(1 / n)$ and $D(n)=\Theta(n)$ under fast mobility model. Obviously, their scheme obtained the same throughput as ours at the cost of much larger delay. The basic idea of their scheme is that, $k$ packets from $k$ different sources will be grouped into one generation, and the relaying scheme is essentially the same as ours. The comparison here raises an interesting question-why in our RLC-based schemes we only mix packets from the same source? The reasons are the following: first of all, as shown in the above comparison, even for all-to-all communication scenarios, mixing packets from different sources is not a good choice. Second, for multiple unicast scenarios, we mix packets from different sources and these packets have different destinations. When one destination decodes a packet designated for another destination, this packet is in fact a duplicate at the first destination which will reduce the throughput. In our multi-hop relay with RLC, we also introduce redundancy for the same reason. However, the redundancy here is explicitly designed for decreasing the delay. While for the former case, it is purely a waste of network resource in multiple unicast scenarios. Finally, grouping packets from different sources requires coordinations. We are not sure about the cost for performing this coordination task, and we are interested in designing fully decentralized schemes, in which the operations from different nodes should be decoupled as much as possible.

\section{Throughrut-Delay Tradeoffs With Network CODING: ANALYSIS}

In this section, we give outlines of proofs for the results on RLC-based relaying schemes discussed in the previous section. Intuitions behind these proofs are also provided.

\section{A. Preliminaries}

To facilitate the theoretical analysis, we need first investigate two critical delays for fast and slow mobility models: minimal delays for 2-hop relays and for flooding. Here, 2-hop relay represents any scheme with controlled redundancy on the number of hops (in the 2-hop relay case, the number of hops for each packet is 2, and other schemes with constant hop constrains will yield the critical delays on the same order of $n$ ), and flooding represents all schemes that remove this constraint totally.

Consider the following situation: initially, only one node's color is red, which we call the source. All other nodes are blue. Whenever a source node encounters a blue node (in the same cell), the latter is colored red. The time for $\Theta(n)$ nodes to become red is called minimal 2-hop delay. If we change the rule slightly: whenever a red node encounters a blue node, the latter is colored red, then the corresponding time is named as minimal flooding delay. Obviously, these two critical delays reflect the intrinsic properties of how mobility will facilitate information propagation. These two quantities 
are scheme-independent, i.e., they hold for any scheme with or without replicas and with or without network coding.

For fast mobility model, the values of these two critical delays are available in the literature [5], and are included here for completeness.

Lemma 1: (Theorem 3 and Lemma 3 in [5]) The minimal 2-hop delay and the minimal flooding delay under fast mobility model are $\Theta(n)$ and $\Theta(\log n)$, respectively.

Next, we present the results for slow mobility model.

Lemma 2: The minimal 2-hop delay under slow mobility model is $\Theta(n)$.

Proof: Under slow mobility model, the joint position of two nodes due to independent random walks can be viewed as a difference random walk relative to the position of one node. Then the inter-meeting times are just the inter-visit times of cell $(1,1)$ for the difference random walk on a $\sqrt{n} \times \sqrt{n}$ torus. Let $\tau$ be the random variable representing the inter-meeting time defined as above. El Gamal et al. prove the following Lemma in [17].

Lemma 3: $\mathbb{E}[\tau]=n$ and $\mathbb{E}\left[\tau^{2}\right]=\Theta\left(n^{2} \log n\right)$.

Let $N$ be the number of distinct nodes the red node has met in $n$ timeslots. Based on above results, we can obtain that $\mathbb{E}[N]=(1-\varepsilon) n$, where $0<\varepsilon<1$ is a constant, and $\sigma_{N}=$ $O(n \log n)$. By Chebyshev inequality, for any $0<\kappa<1$,

$$
\mathbb{P}\{N \leq(1-\kappa) \mathbb{E}[N]\} \leq \frac{\sigma_{N}}{\kappa^{2} \mathbb{E}[N]^{2}}=O\left(\frac{\log n}{n}\right) \rightarrow 0,
$$

which means that $N=\Theta(n)$ w.h.p.

Lemma 4: The minimal flooding delay under slow mobility model is $\Theta(\sqrt{n})$.

Proof: Note that, Theorem 3 already shows that no scheme will obtain a delay better than $\Theta(\sqrt{n})$ under slow mobility model. We need just to show that this is achievable using flooding. We cite the following important result about rumor spreading on torus: Theorem 3 in [44] states that following the flooding rule mentioned above, at timeslot $t$, there exists a sub-torus of size $\sqrt{t} \times \sqrt{t}$, where for each cell in this sub-torus, there exists at least one red node. Therefore, in $\Theta(\sqrt{n})$ timeslots, we can cover the whole torus of size $\sqrt{n} \times \sqrt{n}$ w.h.p.

The following lemma is useful in delay analysis, since it confirms that the effect of transmission scheduling only contributes a constant factor, which can be ignored in asymptotic analysis. Therefore, the time for two desired nodes to meet will dominate the delay of the scheme.

Lemma 5: In the schemes mentioned above, every node will be scheduled to transmit or receive a packet with a constant, non-vanishing probability that is independent of $n$.

Proof: This result can be obtained from Proposition 1. It only depends on the steady state node location distribution. Note that fast and slow mobility models have the same node location distributions in the steady state. Therefore, this result applies to both mobility models.

\section{B. Proof for Main Results}

Proof outline for 2-hop relay with RLC (Theorem 5):

We first prove the case for fast mobility model. Obviously, if we can prove that in $N=\Theta(n)$ timeslots, the destination can receive $\Theta(n)$ coded packets, then based on Proposition 2 the destination has enough coded packets to recover $k=\Theta(n)$ original packets w.h.p. Therefore, delay is upper bounded by $O(n)$ and the throughput is $T(n)=O(1)$.

From the description of the scheme, we know the source will send $m=\Theta(n)$ coded packets to the network. However, the destination may get $k^{\prime}$ packets, which is fewer than $m$ packets for the following reasons:

(1) The source node only delivers coded packets to $m_{1}<m$ different nodes acting as relays in $N$ timeslots;

(2) The Destination node only meets $m_{2}<m_{1}$ relay nodes that have useful information about the generation the destination wants to decode in $N$ timeslots;

(3) Furthermore, $k^{\prime}<m_{2}$ since when the destination meets a relay, it will not always be scheduled to receive a packet from the relay. However, based on Lemma 5, the difference between $k^{\prime}$ and $m_{2}$ caused by the above reasons is upper-bounded by a constant factor and we can assume that $\Theta\left(k^{\prime}\right)=\Theta\left(m_{2}\right)$.

Obviously, if there are $\Theta(n)$ nodes in the network which have useful information for the destination, the probability for the destination to meet a relay in each timeslot is a constant value, i.e., will not scale down with $n$. Therefore, $\mathbb{P}\left\{k^{\prime}=\Theta(n) \mid m_{1}=\Theta(n)\right\}=1$. Thus, we need to prove that $m_{1}=\Theta(n)$, which is established in Lemma 1). Due to space constraints, we do not repeat the proof here.

The proof for the slow mobility case is similar to the argument above, and the differences are given as follows: Recall that Lemma 2 already shows that after $N_{1}=\Theta(n)$ timeslots, $m_{1}=\Theta(n)$. From [45], we know that the mixing time of a simple random walk on a $\sqrt{n} \times \sqrt{n}$ torus is also $\Theta(n)$. Therefore, there exist a constant $\varepsilon$ such that after $N_{2}=\varepsilon n$ timeslots, these $m_{1}$ nodes with coded packets are uniformly distributed in the torus w.h.p. which means that each node in the network has coded packets with a constant probability. Instead of collecting coded packets as soon as possible, the destination nodes begin to collect packets after $N_{1}+N_{2}$ timeslots. It can be proved that the timeslots $N_{3}$ required to collect $n$ coded packets is still $\Theta(n)$. Therefore the total delay $N=N_{1}+N_{2}+N_{3}=\Theta(n)$ w.h.p.

In both mobility models, each source node sends $m=\Theta(n)$ coded packets for each big generation, and each big generation has $\Theta(n)$ original packets, then each coded packet contains $\Theta(1)$ information of original packets. Because every coded packet is transmitted twice, we have $T(n)=\Theta(1)$.

\section{Proof for multi-hop relay with RLC scheme (Theorem 6):}

The central problem here is still the following: can destination node get $\Theta(k)$ coded packets within $\Theta(k)$ timeslots? If it is the case, based on Proposition 2 the destination has enough coded packets to recover $k$ original packets w.h.p. Then the delay is upper bounded by $O(k)$. Replacing $k$ with $\Theta(\log n)$ and $\Theta(\sqrt{n})$ for fast and slow mobility cases, respectively, we obtain the results on delays. Since we get $k$ original packets in $\Theta(k)$ timeslots, the throughput for the phase when the transmissions of this S-D pair dominate the network is $\Theta(1)$. For fairness embedded in the scheme, this situation happens once for $\Omega(1 / n)$ phases. Therefore, longterm throughput $T(n)=O(1)$, which completes the proof.

Next, we concentrate on an equivalent problem: how many timeslots do we need in order to receive at least $\Theta(k)$ coded packets at the destination? We denote it as $N$. Obviously, $\mathbb{E}[N] \leq \mathbb{E}\left[S_{1}\right]+\mathbb{E}\left[S_{2}\right]$, where $S_{1}$ and $S_{2}$ respectively represent the timeslots required for $\Theta(n)$ nodes in the network to have one coded packet for that generation, and the time required for the destination to receive $\Theta(k)$ packets given that other $\Theta(n)$ nodes hold coded packets.

Note that Lemma 1 and Lemma 4 establish that $\mathbb{E}\left[S_{1}\right]=$ $\Theta(\log n)$ and $\mathbb{E}\left[S_{1}\right]=\Theta(\sqrt{n})$ for fast and slow mobility models, respectively. They both agree with $\Theta(k)$ in respective schemes. In fact, it does not happen by coincidence but by 
how we choose the parameter for RLC. Thus we obtain $\mathbb{E}\left[S_{1}\right]=\Theta(k)$. For $S_{2}$, since $\Theta(n)$ nodes in the network have the coded packet, every transmission happened in the destination's cell will help obtain a coded packet w.h.p., which leads to $\mathbb{E}\left[S_{2}\right]=\Theta(k)$. Summing $\mathbb{E}\left[S_{2}\right]$ and $\mathbb{E}\left[S_{2}\right]$ proves the result that $\mathbb{E}[N] \leq \Theta(k)$. See our technical report [43] for detailed proof.

\section{CONCLUDing REMARKS}

In this paper, we characterize the throughput-delay tradeoffs in mobile ad hoc networks (MANETs) with network coding, and compares with the scenarios where only replication and forwarding are allowed in each node. The schemes/protocols achieving those tradeoffs in an effective and decentralized way are proposed and the optimality of the tradeoffs is established. The scenarios in which network coding provides significant improvement on network performance are identified under different node mobility patterns (fast and slow mobility). The insights on when and how information mixing is beneficial for MANETs with multiple unicast and multicast sessions are provided.

\section{REFERENCES}

[1] S. Farrell and V. Cahill, Delay-and Disruption-Tolerant Networking. Boston, MA: Artech House, 2006.

[2] M. Grossglauser and M. D. Tse, "Mobility increases the capacity of ad hoc wireless networks," in Proc. of IEEE INFOCOM 2001, Anchorage, Alaska, April 2001

[3] P. Gupta and P. R. Kumar, "The capacity of wireless networks," IEEE Transactions on Information Theory, vol. 46, no. 2, pp. 388-404, March 2000.

[4] A. E. Gamal, J. Mammen, B. Prabhakar, and D. Shah, "Throughputdelay trade-off in wireless networks," in Proc. of IEEE INFOCOM 2004, Hong Kong, China, Mar. 2004.

[5] M. Neely and E. Modiano, "Capacity and delay tradeoffs for ad-hoc mobile networks," IEEE Transactions on Information Theory, vol. 51, no. 6, pp. 1917-1937, June 2005.

[6] R. Ahlswede, N. Cai, S. R. Li, and R. W. Yeung, "Network information flow," IEEE Transactions on Information Theory, vol. 46, no. 4, pp. 1204-1216, July 2000.

[7] C. Fragouli and E. Soljanin, Network coding applications, Foundations and Trends in Networking. Boston, MA: Now Publishers Inc., 2007.

[8] S. Jaggi, P. A. Chou, and K. Jain, "Low complxity optimal algebraic multicast codes," in Proc. of IEEE International Symposium on Information Theory, Yokohama, Japan, June-July 2003.

[9] T. Ho, R. Koetter, M. Medard, D. R. Karger, and M. Effros, "The benefits of coding over routing in a randomized setting," in Proc. of IEEE International Symposium on Information Theory, Yokohama, Japan, June-July 2003.

[10] J. Herdtner and E. Chong, "Throughput-storage tradeoff in ad hoc networks," in Proc. of IEEE INFOCOM 2005, Miami, FL, Mar. 2005.

[11] S. Kulkarni and P. Viswanath, "A deternimistic approach to throughpu scaling in wireless networks," IEEE Transactions on Information Theory, vol. 50, no. 6, pp. 1041-1049, Jun. 2004.

[12] M. Franceschetti, O. Dousse, D. Tse, and P. Thiran, "Closing the gap in the capacity of wireless networks via percolation theory," IEEE Transactions on Information Theory, vol. 53, no. 3, pp. 1009-1018, March 2007.

[13] N. Bansal and Z. Liu, "Capacity, mobility and delay in wireless ad hoc networks," in Proc. of IEEE INFOCOM 2003, San Francisco CA, March 2003.

[14] X. Lin and N. Shroff, "The fundamental capacity-delay tradeoff in large mobile ad hoc networks," in Proc. of Third Annual Mediterranean Ad Hoc Networking Workshop, Bodrum, Turkey, June 2004

[15] X. Lin, G. Sharma, R. Mazumdar, and N. Shroff, "Degenerate delaycapacity trade-offs in ad hoc networks with brownian mobility," IEEE/ACM Transactions on Networking, vol. 52, no. 6, pp. 2777-2784, June 2006.

[16] S. Toumpis and A. Goldsmith, "Large wireless networks under fading, mobility, and delay constraints," in Proc. of IEEE INFOCOM 2004 , Hong Kong, China, March 2004.

[17] A. E. Gamal, J. Mammen, B. Prabhakar, and D. Shah, "Optimal throughput-delay scaling in wireless networks - part i: the fluid model," IEEE Transactions on Information Theory, vol. 52, no. 6, pp. 25682592, Jun. 2006.

[18] — "Optimal throughput-delay scaling in wireless networks - part ii: Constant-size packets," IEEE Transactions on Information Theory, vol. 52, no. 11, pp. 5111-5116, Nov. 2006.
[19] G. Sharma, R. R. Mazumdar, and N. B. Shroff, "Delay and capacity trade-offs in mobile ad hoc networks: A global perspective," in Proc. of IEEE INFOCOM 2006, Barcelona, Spain, April 2006

[20] L. Ying, S. Yang, and R. Srikant, "Optimal delay-throughput trade-offs in mobile ad hoc networks," to appear in IEEE Transactions on Information Theory, 2008. [Online]. Available: http://www.ece.iastate. edu/ leiying/Publications/IT.pdf

[21] L. Kleinrock and J. A. Silvester, "Optimum transmission radii in packet radio networks or why six is a magic number," in Proc. of the National Telecommunications Conference, Dec. 1978.

[22] T. Spyropoulos, K. Psounis, and C. S. Raghavendra, "Efficient routing in intermittently connected mobile networks: The multi-copy case," IEEE Transaction on Networking, vol. 16, no. 1, pp. 77-90, Feb. 2008.

[23] S.-Y. Li, R. W. Yeung, and N. Cai, "Linear network coding," IEEE Transaction on Information Theory, vol. 49, no. 2, pp. 371-381, Feb. 2003.

24] P. A. Chou, Y. Wu, and K. Jain, "Practical network coding," in Proc. of 41th Annual Allerton Conference on Communication, Control and Computing, Monticello, IL, Oct. 2003.

[25] S. Deb, M. Medard, and C. Choute, "Algebraic gossip: A network coding approach to optimal multiple rumor mongering," IEEE Transactions on Information Theory, vol. 52, no. 6, pp. 2486-2507, June 2006

[26] S. Katti, H. Rahul, W. Hu, D. Katabi, M. Medard, and J. Crowcroft "Xors in the air: Practical wireless network coding," in Proc. of ACM SIGCOMM 2006, Pisa, Italy, Sept. 2006.

[27] D. S. Lun, M. Medard, and R. Koetter, "Efficient operation of wireless packet networks using network coding," in Proc. of International Workshop on Convergent Technologies 2005, Oulu, Finland, June 2005.

[28] C. Adjih, S. Cho, and P. Jacquet, "Near optimal broadcast with network coding in large sensor networks," in First International Workshop on Information Theory for Sensor Netwoks, Santa Fe, NM, June 2007.

[29] C. Fragouli, J. Widmer, and J.-Y. L. Boudec, "On the benefits of network coding for wireless applications," in 4th International Symposium on Modeling and Optimization in Mobile, Ad Hoc and Wireless Networks (WiOpt), Boston, MA, April 2006

[30] _ "Efficient broadcasting using network coding," IEEE Transactions on Networking, vol. 16, no. 2, pp. 450-463, April 2008

[31] Z. Li and B. Li, "Network coding in undirected networks," in Proc. of the 38th Annual Conference on Information Science and Systems (CISS 2004), Princeton, NJ, March 2004.

[32] X. Zhang, G. Neglia, J. Kurose, and D. Towsley, "On the benefits of random linear coding for unicast applications in disruption tolerant networks," in Second Workshop on Network Coding, Theory, and Applications (NetCod), Boston, MA, April 2006.

[33] Y. Lin, B. Li, and B. Liang, "Stochastic analysis of network coding in epidemic routing," IEEE Journal on Selected Areas in Communications, vol. 26, no. 5, pp. 794-808, June 2008.

[34] A. Eryilmaz, A.Ozdaglar, and M. Medard, "On delay performance gains from network coding," in Proc. of the Conference on Information Sciences and Systems, Princeton, NJ, Mar. 2006.

[35] E. Ahmed, A. Eryilmaz, M. Medard, and A. Ozdaglar, "On the scaling law of network coding gains in wireless networks," in Proc. of MILCOM 2007, Orlando, FL, Oct. 2007.

[36] M. Ghaderi, D. Towsley, and J. Kurose, "Reliability benefit of network coding," Tech. Report 07-08, Computer Science Department, University of Massachusetts Amherst, February 2007. [Online]. Available: http://www.ucalgary.ca/ mghaderi/docs/reliability.pdf

[37] J. Liu, D. Goeckel, and D. Towsley, "Throughput order of ad hoc networks employing network coding and broadcasting," in Proc. of Milcom, Washington, DC, Oct. 2006.

[38] — "Bounds on the gain of network coding and broadcasting in wireless networks," in Proc. of INFOCOM 2007, Anchorage, Alaska, May 2007.

[39] A. Keshavarz-Haddadt and R. Riedi, "Bounds on the benefit of network coding: Throughput and energy saving in wireless networks," in Proc. of INFOCOM 2008, Phoenix, AZ, April 2008.

[40] M. Penrose, Random Geometric Graphs. Oxford: Oxford University Press, 2003.

[41] R. Meester and R. Roy, Continuum Percolation. Cambridge: Cambridge University Press, 1996.

[42] O. Dousse, M. Franceschetti, and P. Thiran, "Information theoretic bounds on the throughput scaling of wireless relay networks," in Proc. of IEEE INFOCOM 2005, Miami, FL, Mar. 2005.

[43] C. Zhang, X. Zhu, and Y. Fang, "Throughput-delay tradeoffs in large-scale manets with network coding," Technical Report, April 2008. [Online]. Available: http://winet.ece.ufl.edu/ ysong/files/tech082.pdf

[44] H. Kesten and V. Sidoravicius, "The spread of a rumor or infection in a moving population," Annals of Probability, vol. 33, no. 6, pp. 24022462, 2005 .

[45] D. Aldous and J. Fill, "Reversible markov chains and random walks on graphs," Monograph in preparation. [Online]. Available: http://www.stat.berkeley.edu/ aldous/RWG/book.html 